\title{
Adaptación y validación del Cuestionario Breve de Confianza Situacional en universitarios mexicanos que consumen alcohol
}

\author{
Martha Leticia Salazar Garza', Ma. de los Ángeles Vacio Muro', Juan José Macías Rodríguez² \\ I Departamento de Psicología, Centro de Ciencias Sociales y Humanidades, Benemérita Universidad Autónoma de Aguascalientes, México \\ 2 Departamento de Estadística, Centro de Ciencias Básicas, Benemérita Universidad Autónoma de Aguascalientes, México
}

\section{RESUMEN}

Introducción: la autoeficacia para el control del consumo de alcohol es una variable relevante en los programas de intervención de las adicciones. El Cuestionario Breve de Confianza Situacional (CBCS) fue desarrollado para adultos bebedores problema en Canadá y posteriormente incluido en los programas de intervención para adultos y adolescentes en México, aunque el único dato reportado en el país ha sido su índice de consistencia interna, por lo que se propuso evaluar si el cuestionario contaba con características psicométricas semejantes a las reportadas previamente con adultos canadienses y con universitarios mexicanos. Objetivo: obtener la validez factorial y confiabilidad del CBCS con estudiantes mexicanos. Método: 1,175 universitarios voluntarios, consumidores de alcohol en el último año y que reportaron dos o más indicadores para el trastorno por consumo de alcohol del DSM 5. La evaluación siguió las normas para el desarrollo y revisión de estudios instrumentales. Resultados: el análisis factorial confirmatorio arrojó un modelo final con un índice de consistencia interna de .78 y de una sola dimensión de cuatro reactivos (probando el control sobre el consumo, necesidad física, conflictos con otros y momentos agradables con otros). Discusión y conclusiones: a diferencia de lo encontrado con adultos bebedores problema, en este trabajo se obtuvo un cuestionario con un nivel de confiabilidad aceptable, compuesto por una sola dimensión, posiblemente se debe a las características de la población. El cuestionario puede emplearse en los programas de intervención o en estudios que midan la autoeficacia situacional para el consumo de alcohol en jóvenes universitarios mexicanos.

Palabras clave: consumo de alcohol, estudiantes universitarios, autoeficacia, situaciones de consumo.

\begin{abstract}
Introduction: self-efficacy for the control of alcohol consumption is a relevant variable in addiction intervention programs. The Brief Situational Confidence Questionnaire (CBCS) was developed for adult problem drinkers in Canada and subsequently included in intervention programs for adults and adolescents in Mexico. Although, the only data reported in our country has been its internal consistency index. Therefore, it was proposed to evaluate whether the questionnaire had similar psychometric characteristics, as previously reported with Canadian adults, with Mexican college students. Objective: obtain the validity and reliability of the CBCS with Mexican students. Method: 1,175 university volunteers, alcohol consumers in the last year and who reported two or more indicators for DSM 5 alcohol use disorder. The evaluation followed the standards for the development and review of instrumental studies. Results: the confirmatory factor analysis yielded a final model of a single dimension of four reagents: testing control over consumption, physical need, conflicts with others and pleasant moments with others, with an internal consistency index of .78. Discussion and conclusions: unlike what was found with adult problem drinkers, in this work a questionnaire composed of a single dimension was obtained, possibly due to the characteristics of the population, with an acceptable level of reliability. The questionnaire can be used in intervention programs or in studies that measure the situational self-efficacy for alcohol consumption in Mexican university students.
\end{abstract}

Keywords: alcohol consumption, university students, self-efficacy, consumption situations.

\footnotetext{
Autor de correspondencia:

Martha Leticia Salazar Garza. Departamento de Psicología, Universidad Autónoma de Aguascalientes. Av. Universidad núm. 940, Ciudad Universitaria, 20131, Aguascalientes, Aguascalientes. Correo electrónico: mlsalazar@correo.uaa.mx

Recibido: 17 de julio de 2019

Aceptado: 27 de septiembre de 2019

doi: 10.28931/riiad.2019.2.04
} 


\section{INTRODUCCIÓN}

La autoeficacia es definida como las creencias en las propias capacidades para organizar y ejecutar cursos de acción para manejar situaciones que se enfrenten en el futuro (Bandura, 1999). Esta variable ha sido considerada relevante en los programas de intervención y prevención para las adicciones (Marlatt, Baer, \& Quigley, 2009), dirigidas a poblaciones de usuarios de alcohol y otras drogas. Se ha encontrado una asociación entre la autoeficacia de los individuos con el éxito en el logro de las metas de tratamiento (Ludwig, Tadayon-Manssuri, Strik, \& Moggi, 2013), con una baja habilidad para rechazar el consumo de alcohol y con la predicción del uso de alcohol y otras drogas (Chomsria, Likhitsathian Aramrattana, \& Siviroj, 2018; Hasking, Boyes, \& Mullan, 2015; Uzun \& Kellecci, 2018).

De manera particular, estudios realizados con estudiantes universitarios también han reportado la relación entre bajos niveles de autoeficacia y mayor frecuencia de embriaguez, mayor consumo de alcohol percibido en amigos, resaltando el grupo de los hombres y los de mayor edad con menores niveles de autoeficacia para rechazar el consumo de alcohol. Además, la baja autoeficacia incide en el consumo de quienes tienen una alta búsqueda de sensaciones (Cicognani \& Zani, 2011).

Dado que la variable de autoeficacia juega un papel primordial en la conducta de beber, se han construido instrumentos para su evaluación. Uno de estos instrumentos es el Cuestionario Breve de Confianza Situacional (CBCS), desarrollado como parte de los modelos de prevención de recaídas dirigidos a adultos bebedores problema en Canadá, partiendo del supuesto de que los individuos perciben su capacidad para controlar su consumo de alcohol en diferente medida, a través de distintas situaciones de riesgo para el consumo, tales como estados emocionales positivos o negativos (Breslin, Sobell, Sobell, \& Agrawal, 2000). El CBCS evalúa de manera breve y económica la autoeficacia de los individuos para enfrentar ocho diferentes situaciones de consumo: momentos agradables con otros, emociones agradables, conflictos con otros, necesidad física, probando el control sobre el consumo, emociones desagradables, presión social y malestar físico.

Los análisis iniciales, con una muestra de adultos bebedores problema en Canadá, mostraron una solución factorial de dos dimensiones con un índice de consistencia interna de .85. Uno de los factores integraba situaciones que involucran afecto negativo (emociones desagradables, conflictos con otros, emociones agradables, urgencia y tentación por el consumo y probando el control sobre el consumo), y el otro incluyó situaciones de alto riesgo placenteras y sociales (momentos agrada- bles con otros, emociones placenteras y presión social). Además, en esta evaluación se encontró que el CBCS tuvo correlaciones negativas con los puntajes obtenidos en las mediciones que evaluaban dependencia, es decir, a menor confianza situacional mayor puntaje en dependencia, especialmente en las situaciones de conflictos con otros y probando el control sobre el consumo.

El CBCS fue empleado en México con adultos bebedores problema como parte de modelos de intervención breve para adultos. La traducción del instrumento estuvo a cargo de Ayala y Echeverría en 1997 (citado en Martínez, Salazar, Ruiz, Barrientos, \& Ayala, 2005), quienes tradujeron los reactivos y los evaluaron con una muestra de personas adultas que tenían consumo excesivo de alcohol, encontrándose un nivel de confiabilidad de .97. Posteriormente, se incluyó en los programas de prevención dirigidos para adolescentes que se inician en el consumo de alcohol y otras drogas. La evaluación del cuestionario se realizó con una muestra de 100 adolescentes y se obtuvo un índice de consistencia interna de .97 (Martínez et al., 2005). No obstante, en ambos casos no se reportó la validez factorial del instrumento.

Debido a lo anterior, el CBCS fue propuesto para su uso con estudiantes universitarios en riesgo por su consumo de alcohol, como parte de los proyectos de investigación y los programas de intervención desarrollados en México (Salazar, Pérez, Avila, \& Vacio, 2012). Se consideró necesario evaluar sus características psicométricas con jóvenes universitarios de México que consumen alcohol y presentan consecuencias negativas relacionadas, ya que se planteó que este podrían tener características psicométricas diferentes, debido a que el instrumento fue desarrollado para población adulta de Canadá y con consumo de alcohol problemático. El objetivo de este artículo es reportar los resultados de su adaptación y validación con una muestra de estudiantes mexicanos consumidores de alcohol.

\section{MÉTODO}

\section{Diseño del estudio}

Para este estudio se siguió un diseño de corte transversal con una muestra por conveniencia de 1,175 universitarios mexicanos, quienes cursaban el primer año de su formación académica y aceptaron participar en el estudio de manera voluntaria.

\section{Participantes}

Los estudiantes incluidos en el estudio tenían de 18 a 29 años, con una edad promedio de 19.20 años ( $S D=$ 1.67). $63.1 \%$ fueron hombres y $36.9 \%$ mujeres. $98.4 \%$ 
reportó ser soltero(a) y el porcentaje restante se dividió entre los que dijeron estar casados, divorciados o en unión libre. $76.1 \%$ estudiaba en universidades públicas y $23.9 \%$ en universidades privadas.

\section{Sede donde se llevó a cabo la recolección de datos}

El levantamiento de la información se realizó en cuatro universidades privadas y seis públicas del estado de Aguascalientes, México, asociadas al Consejo Interuniversitario Contra las Adicciones (CICA).

\section{Mediciones}

Aviso de confidencialidad. Documento en el que se le informa al estudiante qué institución está a cargo del proyecto, la persona y el medio de contacto para cualquier duda sobre el estudio o para cancelar su participación, y el uso que se dará a los datos; asimismo, se le garantiza la confidencialidad de la información y representa el consentimiento para participar en la investigación.

Cuestionario de datos sociodemográficos. Recaba información sobre sexo, edad, estado civil, universidad, carrera y semestre/cuatrimestre que cursa el estudiante. También evalúa el consumo de alcohol en los últimos 12 meses y en el último mes. Se elaboró considerando los reactivos de las Encuestas con Estudiantes realizadas en México.

Cuestionario de Confianza Situacional, versión breve (Echeverría y Ayala, 1997, citado en Martínez et al., 2005). Tiene el objetivo de medir el concepto de autoeficacia con relación a la percepción del usuario sobre su habilidad para enfrentar de manera efectiva ocho situaciones de consumo, con una escala de respuesta del 0 al $100 \%$. Este cuestionario tiene una consistencia interna de 0.97 medida a partir del alfa de Cronbach, tanto para población adulta del país, como para adolescentes consumidores de alcohol y otras drogas.

Lista de indicadores del DSM 5 (American Psychiatric Association, 2014). Evalúa los trastornos psiquiátricos por el consumo de alcohol, considerando el historial de consumo en los últimos 12 meses, con una escala de respuesta dicotómica (sí/no). Clasifica la gravedad del consumo actual en: leve (de dos a tres síntomas), moderado (de cuatro a cinco síntomas) y grave (seis o más síntomas). Esta clasificación se hace de acuerdo con el número de indicadores que se responden de manera afirmativa.

\section{Procedimiento}

En primer lugar se solicitó el consentimiento para el uso y adaptación de los reactivos a una de las investiga- doras que hizo la adaptación del Cuestionario Breve de Confianza Situacional con adultos bebedores problema en México, mismo que fue otorgado. Enseguida, se realizó una revisión teórica del concepto de autoeficacia y de la manera de medir la variable, considerando las aportaciones de Schwarzer et al., (2003), por lo que se modificó la escala de respuesta del cuestionario y la redacción de las preguntas. La forma original de obtener las respuestas consistía en que los individuos debían marcar en una escala de porcentaje del 0 al 100 la confianza percibida con la que podían controlar su consumo en diferentes situaciones. La escala se modificó por una de tipo Likert: No confío en mí, Confío poco en mí mismo, Confío mucho en mí mismo y Confío totalmente en mí mismo.

De igual manera, se modificó la redacción de las instrucciones y preguntas. Originalmente, en la versión en español para bebedores problema, se solicitaba a los individuos imaginar que se encontraban en cada situación evaluada y se les solicitaba reportar qué tan seguros se sentían de poder controlar su manera de beber alcohol en exceso, marcando con una X si definitivamente consumiría o definitivamente no consumiría, en una escala del 0 al 100\%. Enseguida, se presentaban las ocho diferentes situaciones que se evalúan: emociones desagradables, malestar físico, emociones agradables, probando el control sobre el consumo de alcohol, necesidad física, conflicto con otros, presión social y momentos agradables con otros. El cambio consistió en dar la instrucción general de marcar con $\mathrm{X}$ la opción de respuesta (No confío en mí, Confío poco en mí mismo, Confío mucho en mí mismo y Confío totalmente en mí mismo) que más se acercaba a la confianza que percibían los universitarios para controlar su manera de beber, seguida por las diferentes situaciones evaluadas, anteponiéndoles el adverbio "incluso", para presuponer una situación hipotética que posiblemente fuera difícil de enfrentar, por ejemplo: "Confío en mi capacidad para controlar mi forma de beber alcohol... Incluso si siento emociones desagradables (por ejemplo: si estuviera deprimido en general, si las cosas estuvieran saliendo mal).

A continuación, se realizó un jueceo por parte de tres jueces expertos en la prevención de adicciones, quienes evaluaron las instrucciones, opciones de respuesta, redacción de las preguntas y formato del instrumento. Los jueces sugirieron modificar el adverbio "incluso" por el de "aun". Una vez atendida la observación de los jueces, se solicitó permiso a las diferentes instituciones participantes para aplicar los instrumentos, acordando el horario más pertinente para los estudiantes. La aplicación se realizó con 5,029 universitarios de manera electrónica en los laboratorios de cómputo asignados para ello, en los que se encontraban dos de los colaboradores del proyecto, quienes explicaban el objetivo de la evaluación a 
Ios alumnos, les solicitaban leer el formato de consentimiento y responder la evaluación, en caso de estar de acuerdo en participar en la investigación. El análisis final se realizó con 1,175 estudiantes, quienes cubrieron los criterios de selección mencionados previamente.

\section{Análisis estadísticos}

Inicialmente se realizó un análisis de los reactivos, a través de la prueba $t$ de Student para muestras independientes, a fin de determinar si estos discriminaban entre el grupo con las calificaciones más altas y el grupo con las calificaciones más bajas, así como un análisis de correlación ítem-total, mediante al alfa de Cronbach.

Los reactivos que pasaron los análisis previos fueron sometidos a la prueba de esfericidad de Barlett y el coeficiente Kaiser-Meyer-Olkin, con el fin de establecer si se justificaba o no el análisis factorial. Enseguida, se corrió un análisis factorial exploratorio de componentes principales, mediante rotación ortogonal, a través del método varimax. Se conservaron los factores que tuvieron autovalores mayores a 1 y reactivos con cargas factoriales iguales o mayores a .40, sin que cargaran en dos o más factores. Posteriormente, a fin de disminuir el error en la medición, se realizó un análisis factorial confirmatorio de máxima verosimilitud, a través del módulo AMOS del SPSS versión 20. Se conservó el modelo que tuviera una Chi-cuadrada igual o menor a 3, un RMSEA menor a .05 y valores mayores a .90 para los índices NFI, RFI, IFI, TLI y CFI. Con los reactivos que se conservaron con el análisis factorial confirmatorio se analizó el índice de consistencia interna mediante el coeficiente alfa de Cronbach.

\section{Consideraciones éticas}

Cada participante firmó un aviso de confidencialidad, en el cual se explicaba la finalidad del estudio, el resguardo y la protección de sus datos; asimismo, se señalaba la importancia de su participación. Además, los estudiantes identificados en mayor riesgo por su manera de beber fueron invitados a realizar una evaluación más amplia sobre su consumo de alcohol, mediante una página web diseñada para ellos (Pérez, Ortiz, Vacio, \& Salazar, 2016).

\section{RESULTADOS}

\section{Características del consumo de alcohol y otras drogas}

Todos los participantes reportaron el consumo de una cerveza o "cuba" de cualquier otra bebida alcohólica alguna vez en la vida. El 100\% reportó consumo de alco- hol en los últimos 12 meses; mientras que $45.7 \%$ dijo haber bebido una copa o cerveza completa de dos a tres veces en el último mes; $35.8 \%$ una o más veces en la última semana; $13.3 \%$ una vez en el último mes; y 5.2\% no bebió en el último mes. Respecto al consumo en el último mes de cinco o más cervezas o cualquier bebida alcohólica en una sola ocasión, $40.3 \%$ señaló haberlo hecho de dos a tres veces; $25.7 \%$ una vez; $20.8 \%$ una o más veces en la última semana; y el 13.3\% restante dijo no haber bebido en esta cantidad en el último mes.

Por otra parte, $54.3 \%$ reportó de dos a tres síntomas en el DSM 5M; $24.7 \%$ de cuatro a cinco síntomas; y $21.0 \%$ reportó seis o más.

\section{Análisis de discriminación de reactivos}

La prueba $t$ de Student para muestras independientes mostró que todos los reactivos discriminaron entre el grupo con las calificaciones más altas y el grupo con las calificaciones más bajas. Además, el índice de correlación reactivo, calificación total, reveló que todos los reactivos obtuvieron un índice mayor a .20, por lo que todos fueron considerados para realizar un primer análisis factorial exploratorio (ver Tabla 1).

\section{Validez factorial}

La prueba de esfericidad de Barlett y el coeficiente Kaiser-Meyer-Olkin evidenciaron una correlación entre los reactivos. La prueba de Barlett mostró que la matriz de la correlación no era idéntica (3932.50; $p=<.000)$. El puntaje Kaiser-Meyer-Olkin alcanzó un valor significativo meritorio (0.887). El análisis factorial exploratorio mostró la presencia de un solo factor, conservando los ocho reactivos con carga factorial mayor a .40, con un autovalor de 4.28 , mismo que explicó el $53.56 \%$ de la varianza total.

No obstante, con el análisis factorial confirmatorio se eliminaron cuatro reactivos (AE1 = Aun si siento emociones desagradables, $\mathrm{AE} 2=$ Aun si siento malestar físico, AE3 = Aun si siento emociones agradables, y AE7 = Aun si sintiera presión social). Los resultados mostraron que el modelo propuesto de cuatro reactivos (ver Figura 1) tuvo el mejor ajuste $X^{2}=.920 \mathrm{gl}=2, p=.398$, RMSEA $=.00, \mathrm{NFI}=.999, \mathrm{RFI}=.996, \mathrm{IFI}=1, \mathrm{TLI}=1, \mathrm{CFI}=1$. El instrumento obtuvo una confiabilidad general de .78.

\section{DISCUSIÓN}

El estudio permitió obtener un cuestionario válido y confiable para estudiantes universitarios mexicanos, integrado por una sola dimensión con cuatro reactivos (ver Tabla 2), lo que difiere de estudios previos realizados 
Tabla 1

Análisis de discriminación de reactivos y correlación ítem-total

\begin{tabular}{|c|c|c|c|}
\hline \multirow[t]{2}{*}{ Reactivos } & \multicolumn{2}{|c|}{$\begin{array}{l}\text { Prueba t para la } \\
\text { igualdad de medidas }\end{array}$} & \multirow{2}{*}{$\begin{array}{c}\text { Correlación } \\
\text { reactivo- } \\
\text { calificación } \\
\text { total }\end{array}$} \\
\hline & $t$ & $\begin{array}{l}\text { Sig. } \\
\text { (bilateral) }\end{array}$ & \\
\hline $\begin{array}{l}\text { AE1. Aun si siento emociones desagradables (por ejemplo: si estuviera } \\
\text { deprimido en general, si las cosas estuvieran saliendo mal). }\end{array}$ & -33.266 & .000 & .646 \\
\hline $\begin{array}{l}\text { AE2. Aun si siento algún malestar físico (por ejemplo: si no pudiera dormir, si me } \\
\text { sintiera nervioso y tenso). }\end{array}$ & -25.742 & .000 & .609 \\
\hline $\begin{array}{l}\text { AE3. Aun si siento emociones agradables (por ejemplo: si me sintiera muy } \\
\text { contento, si quisiera celebrar, si todo fuera por buen camino). }\end{array}$ & -26.715 & .000 & .582 \\
\hline $\begin{array}{l}\text { AE4. Aun si quisiera probar mi control sobre el consumo de alcohol (por ejemplo: } \\
\text { si pienso que puedo detenerme o dejar de tomar cuando yo lo decida). }\end{array}$ & -26.279 & .000 & .655 \\
\hline $\begin{array}{l}\text { AE5. Aun si siento necesidad física (por ejemplo: si tuviera urgencia de beber, si } \\
\text { pensara "que rico es beber alcohol"). }\end{array}$ & -31.695 & .000 & .691 \\
\hline $\begin{array}{l}\text { AE6. Aun si tengo conflictos con otros (por ejemplo: si tuviera una pelea con } \\
\text { alguien cercano o importante para mí). }\end{array}$ & -31.962 & .000 & .664 \\
\hline $\begin{array}{l}\text { AE7. Aun si sintiera presión social (por ejemplo: si alguien me presionara para } \\
\text { beber alcohol, si alguien me invitara a su casa para beber una copa). }\end{array}$ & -32.415 & .000 & .631 \\
\hline $\begin{array}{l}\text { AE8. Aun en momentos agradables con otros (por ejemplo: si quisiera celebrar } \\
\text { con un amigo, si me estuviera divirtiendo con un amigo o amiga y quisiera } \\
\text { sentirme mejor). }\end{array}$ & -31.139 & .000 & .600 \\
\hline
\end{tabular}

Nota: muestra los valores ty su significancia estadística, así como los índices de correlación de cada reactivo contra el resto de la escala.

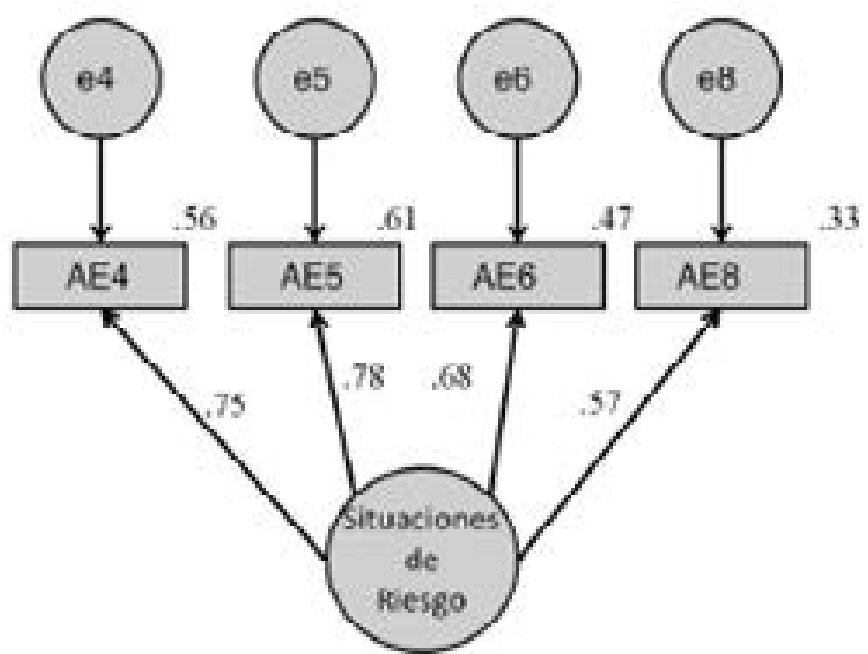

Figura 1. Factorial Confirmatorio. Chicuadrada $=0.920, g l=2, p=$ $.398 \backslash \mathrm{NFI}=.999 \backslash \mathrm{RFI}=.996 \backslash \mathrm{IFI}=|\backslash \mathrm{TLI}=| \backslash \mathrm{CFI}=\mid \backslash \mathrm{RMSEA}=$ .000. AE4 = Aun si quisiera probar mi control sobre el consumo de alcohol. AE5 = Aun si siento necesidad física. AE6 = Aún si tengo conflictos con otros. AE8 = Aun en momentos agradables con otros. 
Tabla 2

Cuestionario Breve de Confianza Situacional para Jóvenes Universitarios Mexicanos

INSTRUCCIONES:

Marca con una X la opción que más se acerque a la confianza que posees para controlar tu forma de beber en cada una de las situaciones que se te muestran a continuación.

\begin{tabular}{|c|c|c|c|c|}
\hline $\begin{array}{l}\text { CONFÍO EN MI CAPACIDAD PARA } \\
\text { CONTROLAR MI FORMA DE BEBER } \\
\text { ALCOHOL... }\end{array}$ & $\begin{array}{c}\text { No confío } \\
\text { en mí mismo }\end{array}$ & $\begin{array}{l}\text { Confío poco } \\
\text { en mí mismo }\end{array}$ & $\begin{array}{l}\text { Confío mucho } \\
\text { en mí mismo }\end{array}$ & $\begin{array}{c}\text { Confío totalmente } \\
\text { en mí mismo }\end{array}$ \\
\hline
\end{tabular}

1. Aun si quisiera probar mi control sobre el consumo de alcohol.

(Por ejemplo: si pienso que puedo detenerme o dejar de tomar cuando yo lo decida).

\section{Aun si siento necesidad física.}

(Por ejemplo: si tuviera urgencia de beber, si pensara "que rico es beber alcohol").

3. Aun si tengo conflictos con otros. (Por ejemplo: si tuviera una pelea con alguien cercano o importante para mí).

4. Aun en momentos agradables con otros. (Por ejemplo: si quisiera celebrar con un amigo, si me estuviera divirtiendo con un amigo o amiga y quisiera sentirme mejor).

Nota: muestra la versión final del cuestionario.

con adultos bebedores problema de Canadá (Breslin et al., 2000). No obstante, los resultados del análisis factorial no pueden compararse con adultos y adolescentes mexicanos, ya que el único dato de su adaptación es el índice de consistencia interna. Si bien dicho índice fue mayor a la versión final encontrada en este estudio, se carece de información que permita comprobar que los ocho reactivos son válidos para población adulta y de adolescentes mexicanos.

La eliminación de cuatro reactivos posiblemente se debe a las características de la población con la cual fue evaluado el instrumento en este estudio. El trabajo realizado en Canadá incluyó adultos con un puntaje de dependencia alto, lo que posiblemente podría haber afectado su autoeficacia en un mayor número de situaciones de consumo (Breslin et al., 2000). En contraparte, los universitarios evaluados en este estudio reportaron beber en cantidades excesivas (cinco o más cervezas o tragos) en el último mes, pero menos de la cuarta parte tuvo un consumo de alcohol grave, con base en el número de indicadores afirmativos del DSM 5.

Dos de los reactivos eliminados hacen referencia al afecto negativo (emociones desagradables y malestar físico) y dos al afecto positivo o placentero (emociones agradables y presión social). La eliminación del reactivo de emociones desagradables posiblemente se deba a que éstas pueden integrarse con la ocurrencia de conflictos interpersonales, situación que puede reflejar en mayor medida el consumo de los estudiantes universitarios evaluados y que permaneció al final del análisis. Por otro lado, el consumo de alcohol ante una situación de malestar físico posiblemente no está asociado entre los jóvenes como una manera de resolver los síntomas derivados de cualquier enfermedad, como lo está para adultos con consumo problemático. Mientras que las emociones agradables probablemente se asocian con la convivencia o momentos de diversión con otros, por lo que este reactivo pudo ser eliminado. Finalmente, el reactivo que evaluaba la presión social como una situación para el consumo pudo haber sido eliminado debido a que los jóvenes no perciban como presión social la insistencia de sus amigos para beber alcohol.

Por su parte, los reactivos que permanecieron en la evaluación final en su mayoría aluden al afecto negativo (probando el control sobre el consumo, necesidad física y conflictos con otros) y uno solo al afecto positivo o pla- 
centero (momentos agradables con otros), lo que posiblemente muestre que son estas las situaciones que, en México, se relacionan en mayor medida con el consumo de los jóvenes universitarios. Esto, a su vez, muestra la importancia de incluir la enseñanza de estrategias para enfrentar dichas situaciones en los programas de atención dirigidos a esta población.

Respecto a lo anterior, es importante resaltar que algunos estudios realizados con jóvenes universitarios de otros países muestran que una mayor labilidad al afecto negativo se asocia con un mayor porcentaje de días de consumo fuerte de alcohol (Weiss, Bold, Contractor, Sullivan, Armeli, \& Tennen, 2018), siendo incluso una variable mediadora entre la exposición al trauma y el consumo de esta sustancia. Por tanto, incluir el manejo del afecto negativo en los tratamientos podría favorecer la disminución del consumo de alcohol y otras drogas en jóvenes universitarios.

Una de las limitaciones del estudio es que sólo se incluyó una muestra de estudiantes universitarios del primer año de estudios. Sería importante realizar los análisis considerando un rango de edad más amplio entre este sector para identificar si existe alguna diferencia con el paso del nivel de estudios y la autoeficacia de los estudiantes para enfrentar las diferentes situaciones de consumo. Además, se podrían evaluar otras evidencias de la validez del instrumento, como su asociación con la búsqueda de sensaciones, ya que estudios previos han mostrado una asociación entre una baja autoeficacia y un mayor puntaje con esta variable (Cicognani \& Zani, 2011; Uzun \& Kellecci, 2018).

En conclusión, el instrumento obtenido puede ser empleado en los programas de intervención con jóvenes universitarios para medir su autoeficacia al inicio, al término y durante la fase de seguimiento de los programas, como una manera de medir su eficacia y asociación con otras variables.

Por otro lado, de manera específica, las situaciones de riesgo identificadas pueden ayudar a mejorar los programas preventivos, centrando sus esfuerzos en la enseñanza de habilidades específicas para enfrentar dichas situaciones. Por ejemplo, se puede proponer la generación de estrategias alternativas para divertirse en los momentos agradables, además de enseñar estrategias para el consumo moderado de alcohol en dichas situaciones; la enseñanza de estrategias para expresar las emociones desagradables o para negociar cuando surgen conflictos en las relaciones interpersonales. También, enseñar a los individuos a identificar los estímulos externos o internos que disparan su deseo por el consumo de alcohol, así como la enseñanza de estrategias que permitan controlar el consumo cuando se ponen a prueba para beber menos.

\section{FUENTES DE FINANCIAMIENTO}

Benemérita Universidad Autónoma de Aguascalientes. Proyecto PIPS14-1, Desarrollo de estrategias para promover la búsqueda de ayuda en jóvenes universitarios que consumen alcohol en exceso.

\section{CONFLICTOS DE INTERÉS}

No hay conflictos de intereses.

\section{AGRADECIMIENTOS}

Los autores agradecen a la Benemérita Universidad Autónoma de Aguascalientes, por el financiamiento otorgado; a los estudiantes de pregrado y posgrado que colaboraron en el estudio; a los participantes y las escuelas de educación superior asociadas al Consejo Interuniversitario Contra las Adicciones del Estado de Aguascalientes (CICA), por las facilidades otorgadas para la realización del estudio; así como a los investigadores que previamente adaptaron el Cuestionario Breve de Confianza Situacional con bebedores problema de México, por su consentimiento para el uso de los reactivos. Por otra parte, se agradece a la Lic. Leticia Echeverría San Vicente, por otorgar el permiso para emplear los reactivos del Cuestionario Breve de Confianza Situacional, a fin de obtener sus características psicométricas con la población incluida en este estudio.

\section{REFERENCIAS}

American Psychiatric Association (2014). DSM 5. Manual Diagnóstico y Estadístico de los Trastornos Mentales. $5 a$ edición. EE. UU: Panamericana.

Bandura, A. (1999). A Social Cognitive Theory of Personality. En: L. Pervin, \& O. John, (Eds.), Handbook of personality (pp. 154196). New York: Guilford Publications.

Breslin, F. C., Sobell, L. C., Sobell, M. B., \& Agrawal, S. (2000). A comparison of a brief and long version of the Situational Confidence Questionnaire. Behaviour Research and Therapy, 38, 1211-1220. doi: 10.1016/s0005-7967(99)00152-7

Chomsria, P., Likhitsathian, S., Aramrattana, A., \& Siviroj, P. (2018). Self-efficacy, sensation seeking, right attitude, and readiness to change among alcohol drinkers in a Thai vocational school. Addictive Behaviors Reports, 8, 107-112. doi:10.1016/j. abrep.2018.08.006

Cicognani, E., \& Zani, B. (2011). Alcohol Use Among Italian University Students: The Role Of Sensation Seeking, Peer Group Norms and Self-Efficacy. Journal of Alcohol and Drug Education, 55(2), 17-36.

Hasking, P., Boyes, M., \& Mullan, B. (2015). Reward and Cognition: Integrating Reinforcement Sensitivity Theory and Social Cognitive 
Theory to Predict Drinking Behavior. Substance Use \& Misuse, 50(10), 1316-1324. doi: 10.3109/10826084.2015.1005315

Ludwig, F., Tadayon-Manssuri, E., Strik, W., \& Moggi, F. (2013). Self-Efficacy as a Predictor of Outcome After Residential Treatment Programs for Alcohol Dependence: Simply Ask the Patient One Question! Alcoholism: Clinical and Experimental Research, 37(4), 663-667. doi: 10.1111/acer.12007

Marlatt, G. A., Baer, J. S., \& Quigley, L. A. (2009). Selff-efficacy and addictive behavior. In A. Bandura, (Ed.) Self-efficacy in Changing Societies. (pp. 289-316). Cambridge: Cambridge University Press.

Martínez, M. K. I., Salazar, G. M. L., Ruiz, T. G. M., Barrientos, C. V., \& Ayala, V. H. E. (2005). Programa de intervención breve para adolescentes que inician el consumo de alcohol y otras drogas. Manual del terapeuta. México, D.F.: Universidad Nacional Autónoma de México, Facultad de Psicología.

Pérez, C. J. L., Ortiz, N. S. J., Vacio, M. M. A., \& Salazar, G. M. L. (2016). Percepción de una intervención en modalidad electró- nica por universitarios que consumen alcohol. Caleidoscopio, 34, 77-93.

Salazar, G. M., Pérez, C. J., Avila, H. O., \& Vacio, M. M. A. (2012). Consejo breve a universitarios que consumen alcohol en exceso: resultados iniciales. Psicología y Salud, 22(2), 247-256.

Schwarzer, R., Sniehotta, F. F., Lippke, S., Luszczynska, A., Scholz, U., Schüz, B., ... Ziegelmann J. P. (2003). On the Assessment and Analysis of Variables in the Health Action Process Approach: Conducting an Investigation. Berlin: Freie Universität Berlin.

Uzun, Z., \& Kellecci, M. (2018). Substance Abuse in High School Students: Their Self-Efficacy to Avoid Substance Abuse and Related Factors. The Journal of Psychiatry and Neurological Sciences, 31(4), 856-868. doi: 10.5350/DAJPN2018310404

Weiss, N. H., Bold, K. W., Contractor, A. A., Sullivan, T. P., Armeli, S., \& Tennen, H. (2018). Trauma exposure and heavy drinking and drug use among college students:Identifying the roles of negative and positive affect lability in a daily diarystudy. Addictive Behaviors, 79, 131-137. doi: 10.1016/j.addbeh.2017.12.015 